\title{
Mutation: In spike protein of SARS-CoV-2 Variant
}

\author{
Jitendra Kumar' ${ }^{1}$, Gauttam Bhatia ${ }^{2}$, Arpeeta Mazumdar ${ }^{3}$, Maneesh \\ Bagai $^{4}$ and Ashok Rattan ${ }^{5 *}$ \\ ${ }^{1}$ Scientist, Molecular Biology, Pathkind Labs, Gurgaon, India \\ ${ }^{2}$ Consultant Pathologist, Pathkind Labs, Gurgaon, India \\ ${ }^{3}$ Consultant Microbiologist, Pathkind Labs, Gurgaon, India \\ ${ }^{4}$ Director, Regional Reference Laboratory, Pathkind Labs, Gurgaon, India \\ ${ }^{5}$ Advisor, Pathkind Labs, Gurgaon, India \\ *Corresponding Author: Ashok Rattan, Advisor, Pathkind Labs, Gurgaon, India. \\ DOI: $10.31080 /$ ASMI.2020.04.0767
}

\section{Background of Coronavirus}

On $31^{\text {st }}$ December 2019, the World Health Organization (WHO) documented a novel coronavirus that causes severe acute respiratory syndrome 2 (SARS-CoV-2) [1]. The pilot case started from Wuhan, China and got spread globally via transmission through the person to person contact. Coronavirus is a single stranded RNA virus. Its length is 26 to 36 kilo-bases. As per nomenclature, it belongs to subfamily Coronavirinae, family Coronaviridae, order Nidovirales. On the basis of genomic and serological changes, Alphacoronavirus, Betacoronavirus, Gammacoronavirus and Deltacoronavirus are the four major genera of subfamily Coronavirinae [2]. In humans, six types of coronaviruses are reported viz HCoV-NL63, HCoV-229E, HCoV-OC43, HCoVHKU1, SARS-CoV (The severe acute respiratory syndrome coronavirus), and MERS-CoV (The Middle East respiratory syndrome coronavirus). As a result of human-animal interface activities, large genetic diversity with frequent recombination of genomes, different variations can occur in coronaviruses [3-5].

Summary of new variant

Within a year of inception of the pandemic, in December 2020, a new variant strain of severe acute respiratory syndrome was found named as B.1.1.7. It was first noticed in Kent and Greater London and then eventually spread to South East England along with other locations in the United Kingdom (UK). Other countries included are Wales (20 cases), Denmark ( 9 cases), Netherland (1 case), Australia (1 case). These were identified with the help of GIASAID EpiCov database [6,7]. As per the media, four cases were also reported in Belgium.

On the basis of genome sequence data of virus, it was identified that a large proportion of such cases belonged to a new single phylogenetic cluster. The new variant is more transmissible and spreading rapidly than previously circulating strains as per COGUK (COVID-19 Genomics U.K. Consortium). COG-UK Consortium reported that it added 2,963 more genome sequences of SARSCoV-2. As per the present studies, these variants are predominating in some geographical regions that does not mean that it is more infectious. For our better understanding about the new variants, scientists/doctors are working and trying to understand its cause, infectious rate, transmission rate and effect on present vaccines. In the present scenario, we cannot comment or conclude about the new variant SARS-CoV-2 illness, risk of death, virology, epidemiologic and clinical characteristics $[8,9]$.

Nomenclature of new variant

On the basis of different concerning epidemiological, immunological or pathogenic properties of SARS-CoV-2, they are designated Variant Under Investigation (VUI) name for the new SARSCoV-2 variant in the UK with a year, month, and number. Finally, expert committee designated it as Variant of Concern (VOC). Now it is referred as "SARS-CoV-2 VOC 202012/01" (i.e., the first variant of concern from 2020, December) or "B.1.1.7." on 18/12/2020 $[6,7,10,11]$.

\section{Mutations in new variant of SARS-CoV-2}

This SARS-CoV-2 virus new variant VOC 202012/01 is defined by 23 mutations: 13-non synonymous (codon translate the different amino acid) mutations, 4 deletions and 6 synonymous mutations (codon translate the same amino acid) or silent mutation (means no changes in the structure of the proteins). The non-synonymous mutations include a series of spike protein mutations.

B.1.1.7 has an unusually large number of genetic changes, especially in the spike protein. Three of them have potential biological 
effects [7]. It is reported that N501Y has more binding affinity with human ACE2 (Angiotensin-converting enzyme 2) in the receptor binding domain (RBD) of the spike protein at position 501, where amino acid asparagine $(\mathrm{N})$ has been replaced with tyrosine $(\mathrm{Y})$. It is also named as S:N501Y. The spike deletion 69-70 has also occurred a number of times with other RBD changes. It leads to change in the shape of the spike protein. Mutation $\mathrm{P} 681 \mathrm{H}$ is immediately adjacent to the furin cleavage site, a site with high unevenness in coronaviruses and known location of biological importance. Apart from multiple spike protein mutations (A570D, D614G, T716I, S982A, $\mathrm{D} 1118 \mathrm{H})$, some other mutations are also reported in genomic regions $[6,7,12]$.

Other one mutation reported in a stop codon open reading frame 8 (ORF8) i.e. not in the spike protein. It is in a different gene that's function is not known [6,7]. Reported 6 synonymous mutations are, 5 in ORF1ab (C913T, C5986T, C14676T, C15279T, C16176T) and one in the M gene (T26801C) [7].

Impact of spike variant S: N501Y

- Transmissibility: Transmissibility rate is up to $70 \%$. It is reported that, one mutation D614G spreads faster than the viruses without mutation in the human respiratory epithelial cells. N501Y alone or with the 69/70 deletion mutation is enhancing the transmissibility rate of the virus $[6,8,13]$.

- Illness: There is no report generated on the ability to cause disease in the humans from SARS-CoV-2 new variant strain [7].

- Impact on vaccine: FDA- authorized "Polyclonal" vaccines produce antibodies and target many regions of the spike protein. Due to mutation in the spike protein of the virus, potential impact of the vaccines is affected [7].

- Antigenicity: On the basis of different studies of therapeutic agents like monoclonal antibodies test, due to mutation at position 501 one (LYCoV016) indicated decreased capability to neutralise SARS-CoV-2. From polyclonal sera, no more study is available on N501Y [6].

Impact on diagnostic test

A corona virus contains four main structural proteins including spike (S), membrane (M), envelope (E), and nucleocapsid (N) proteins that are encoded by ORFs. Next-generation segeuencing (NGS) or real- time reverse transcriptase- polymerase chain reaction (RT-PCR) techniques are used for the isolation of the causal agent and to identify the genome sequence of the corona virus [14-16]. This virus is identified from lower respiratory specimen and diagnosis is done by RT-PCR method on the basis of RNA-dependent RNA polymerase (RdRp) gene of the ORF1ab sequence, $\mathrm{E}$ gene, $\mathrm{N}$ gene, and $\mathrm{S}$ gene of the SARS-CoV-2 genome. In the $\mathrm{S}$ gene of VOC there are six nucleotides deletion, due to which double deletion at the position of $69 / 70$ in the spike protein in the new variant gives a negative result from S-gene (S-gene target failure- SGTF) in RT-PCR test [6-8,15-17].

\section{Summary}

A new variant of the SARS-CoV-2 has been reported in few countries and its transmissibility rate is more in comparison to other previous reported viruses. Its ability to cause disease is not clear. Due to multiple mutations in the spike protein, we cannot rely by using the $\mathrm{S}$ gene target on primary detection for diagnosis of new variant strain infection. Role of NGS might be better option to understand the genetic evolution of SAR-CoV-2 and its impact on the antigenic properties and transmissibility of the variant. We can monitor the evolution with the help of sequencing data of the virus. More studies and genome sequencing are needed in this direction for better understanding about the new variant of "SARS-CoV-2 VOC 202012/01".

\section{Bibliography}

1. Amit Pruthi., et al. "How Reliable is your Covid 19 tests? Need to validate and verify Covid 19 tests in the laboratory before clinical use". Acta Scientific Microbiology 3 (2020): 10.

2. Huihui Wang., et al. "The genetic sequence, origin, and diagnosis of SARS-CoV-2". European Journal of Clinical Microbiology and Infectious Diseases (2020) 39:1629-1635.

3. Weiss SR and Leibowitz JL. "Coronavirus pathogenesis". Advances in Virus Research 81 (2011): 85-164. 
4. Li F. "Structure, function, and evolution of coronavirus spike proteins". Annual Review of Virology 3 (2016): 237-261.

5. Tang Q., et al. "Inferring the hosts of coronavirus using dual statistical models based on nucleotide composition". Scientific Report 5 (2015): 17155.

6. Implications of the Emerging SARS-CoV-2 variant VOC 202012/01. Centre for disease control and prevention 22 December (2020).

7. Investigation of novel SARS-CoV-2 variant, variant of concern 202012/01. Public Health England. 21 December (2020).

8. Rapid increase of a SARS-CoV-2 variant with multiple spike protein mutations observed in the United Kingdom. European Centre for Disease Prevention and Control 20 December (2020).

9. COVID-19 Genomics UK Consortium (COG-UK) (2020).

10. SARS-CoV-2 lineages (2020).

11. Rambaut A., et al. "A dynamic nomenclature proposal for SARS-CoV-2 lineages to assist genomic epidemiology". Nature Microbiology 5 (2020): 1403-1407.

12. Andrew Rambaut., et al. "Preliminary genomic characterisation of an emergent SARS-CoV-2 lineage in the UK defined by a novel set of spike mutations: COVID-19 genomics". UK consortium (2020).

13. Volz E., et al. "Evaluating the effects of SARS-CoV-2 Spike Mutation D614G on Transmissibility and Pathogenicity". Cell (2020).

14. Ashok Rattan and Jitendra Kumar. "Clinical Utility of Ct Value in Covid-19 Infection". Acta Scientific Microbiology 3 (2020): 12.

15. Lelia Mousavizadeh and Sorayya Ghasemi. "Genotype and phenotype of COVID- 19: Their roles in pathogenesis". Journal of Microbiology, Immunology and Infection (2020).
16. Alireza Tahamtana and Abdollah Ardebilib. "Real-time RT-PCR in COVID-19 detection: issues affecting the results". Expert Review of Molecular Diagnostics (2020).

17. Patrick C Y., et al. "Coronavirus Genomics and Bioinformatics Analysis". Viruses 2 (2010): 1804-1820.

\section{Assets from publication with us}

- Prompt Acknowledgement after receiving the article

- Thorough Double blinded peer review

- Rapid Publication

- Issue of Publication Certificate

- High visibility of your Published work

Website: www.actascientific.com/

Submit Article: www.actascientific.com/submission.php

Email us: editor@actascientific.com

Contact us: +919182824667 